\title{
Organización vital de una muestra clínica que demanda atención psicológica*
}

\author{
Life Functioning among a Clinical Sample \\ Demanding Psychological Treatment
}

Recibido: 18 de agosto de 2012 | Revisado: 31 de marzo de 2013 | Aceptado: 12 de septiembre de 2013

\author{
FRANCISCO J. LABRADOR ** \\ GLORIA GARCÍA-FERNÁNDEZ \\ MÓNICA BERNALDO-DE-QUIRÓS \\ Departamento de Psicología Clínica, \\ Universidad Complutense de Madrid \\ ROBERTO GÓMEZ \\ CRISTINA MÉNDEZ \\ IGNACIO FERNÁNDEZ-ARIAS \\ Clínica Universitaria de Psicología, \\ Universidad Complutense de Madrid
}

doi: 10.11144/Javeriana.upsy14-1.ovmc

Para citar este artículo: Labrador, F. J., GarcíaFernández, G., Bernaldo-de-Quirós, M., Gómez, R., Méndez, C., \& Fernández-Arias, I. (2015). Organización vital de una muestra clínica que demanda atención psicológica. Universitas Psychologica, 14(1), 381-392. http://dx.doi.org/10.11144/ Javeriana.upsy14-1.ovmc

* Artículo original resultado de investigación

** Correspondencia: Dr. D. Francisco Javier Labrador. Dpto. de Personalidad, Evaluación y Tratamientos Psicológicos I. Facultad de Psicología. Universidad Complutense de Madrid. Campus de Somosaguas. 28223 Madrid.Correo electrónico: labrador@cop.es

\section{RES UMEN}

La asociación entre trastornos psicopatológicos y variables de funcionamiento psicosocial ha sido documentada, pero son escasos los estudios que evalúan su impacto sobre la eficacia de los tratamientos psicológicos. El objetivo de este estudio fue evaluar la organización vital de 78 pacientes de un servicio de atención psicológica y examinar la relación con variables clínicas y con el éxito del tratamiento. Esta organización se evaluó mediante una escala confeccionada ad hoc para este estudio. El área de pareja resultó la menos organizada frente a la familiar y personal, con las mayores puntuaciones. La organización vital se relacionó de forma significativa con distintos ejes del DSM-IV-TR. Se confirmó que pacientes con baja organización vital requieren un mayor número de objetivos terapéuticos.

Palabras clave

funcionamiento psicosocial; tratamiento ambulatorio; evaluación de necesidades; plan de tratamiento; eficacia

\section{A B S T R A C T}

The association between psychological disorders and psychosocial functioning variables has been previously documented, but relatively few studies have examined the impact of these variables on psychological treatment outcomes. The aim of this study was to assess the life functioning of 78 patients from a psychological service centre, and to examine its relationship with clinical variables and treatment success. Life functioning was evaluated with a scale elaborated ad hoc for this study. The partner area of functioning was the most impaired while family and personal areas obtained the highest functioning scores. Patient's life functioning was significantly associated with several axis of DSM-IV-TR. It was confirmed that patients with poorer life functioning required a greater number of treatment objectives.

Keywords

psychosocial functioning; ambulatory care; needs assessment; treatment planning; clinical effectiveness 
Francisco J. Labrador, Gloria García-Fernández, Mónica Bernaldo-de-Quirós,

Roberto Gómez, Cristina Méndez, Ignacio Fernández-Arias

\section{Introducción}

En el ámbito de la atención psicológica, la investigación se ha centrado tradicionalmente en la identificación de los factores más específicos y directamente relacionados con el diagnóstico clínico, en especial síntomas específicos, para la toma de decisiones clínicas. Los avances en esta dirección no siempre han ido acompañados de tasas superiores de éxito de los tratamientos o de un aumento en la calidad de vida de los pacientes, lo que alerta sobre la necesidad de tener en cuenta otros factores entre los que destacan las variables de funcionamiento psicosocial (Fava, Rafanelli, \& Tomba, 2012; Lam, Filteau, \& Milev, 2011; McKnight \& Kashdan, 2009; Rafanelli \& Ruini, 2012).

La asociación entre la incidencia y/o prevalencia de problemas de salud mental y variables socioeconómicas, de trabajo y de salud ha sido documentada, pero no se ha analizado extensamente el impacto de una mínima organización personal previa sobre la eficacia de los tratamientos psicológicos (Alonso \& Lepine, 2007; Bones Rocha, Pérez, Rodríguez-Sanz, Borrell, \& Obiols, 2010; Haro et al., 2006). En la mayoría de los estudios publicados, se examina la influencia de un limitado número de variables psicosociales sobre el tratamiento de trastornos como las adicciones (Sánchez-Hervás et al., 2012; Tracy et al., 2012), la depresión (McKnight \& Kashdan, 2009) o la esquizofrenia (Niv, Cohen, Sullivan, \& Young, 2007; Ritsner, Arbitman, Lisker, \& Ponizovsky, 2012; Smith et al., 2011).

En general, pues, se ha dado poca relevancia a la organización vital (OV) como factor pronóstico de las intervenciones psicológicas, al menos de forma explícita, y no se ha extendido el uso de instrumentos para una evaluación cuantificada del funcionamiento psicosocial. En el eje IV del DSMIV-TR (American Psychiatric Association [APA], 2000) se registran los problemas psicosociales y ambientales que pueden afectar el diagnóstico, el tratamiento y el pronóstico de los trastornos mentales, y en el eje $\mathrm{V}$ se propone la Escala de Evaluación de la Actividad Global (EEAG) para estimar cuál es el nivel de actividad global de los pacientes. Sin embargo, es un índice poco preciso que evalúa únicamente la actividad psicosocial, social y laboral del paciente, y que además, en caso de indicar una desorganización importante no señala en qué ámbitos específicos y cómo cuantificarlos (Dimsdale, Jeste, \& Patterson, 2010; Goldman, Skodol, \& Lave, 1992; Smith et al., 2011). De este modo, se han desarrollado otras escalas complementarias a la EEAG denominadas escala GARF (del inglés Global Assessment of Relational Functioning) y Escala de Evaluación del Funcionamiento Social y Ocupacional (SOFAS) (Hilsenroth et al., 2000) y otras versiones del eje V (Mundo, Bonalume, Del Corno, Madeddu, \& Lang, 2010; Niv et al., 2007), aunque su aplicación apenas se ha diseminado en la práctica clínica habitual.

No obstante, se han desarrollado múltiples instrumentos de evaluación de calidad de vida, tanto genéricos como específicos (Velarde-Jurado \& Ávila-Figueroa, 2002a, 2002b). Los instrumentos de calidad de vida genéricos, entre los que destaca el "grupo SF" (Rebollo, 2008) y el "grupo WHOQOL" de la Organización Mundial de la Salud (Lucas-Carrasco, 2012), tienen una finalidad descriptiva y son útiles para comparar diferentes poblaciones clínicas y monitorizar la salud poblacional, pero pueden resultar poco sensibles a los cambios clínicos. Los instrumentos específicos se basan en las características específicas de trastornos psicopatológicos y médicos y se dirigen a evaluar la evolución y los efectos del tratamiento. Estos instrumentos se han aplicado especialmente para la evaluación de la calidad de vida de población que presenta enfermedades médicas (Silveira, Kern, \& Chem, 2012; Verdonck-de Leeuw, van Nieuwenhuizen, \& Leemans, 2012) y en el ámbito de la salud mental, para población con trastornos mentales crónicos (Morosini, Magliano, Brambilla, Ugolini, \& Pioli, 2000; García-Portilla et al., 2011) y adicciones (Lozano et al., 2008). Sin embargo, y a pesar de las variadas aplicaciones de estos instrumentos, el constructo calidad de vida ha sido considerado en algunas ocasiones como un paraguas conceptual (Urzúa \& Caqueo-Urízar, 2012). 
La OV podría definirse como 'el nivel en que una persona está integrada y disfruta de forma adaptativa en las áreas de funcionamiento personal, de pareja, familiar, laboral y social'. La importancia de la OV radica, entre otros aspectos, en que supone la base sobre la que instalar nuevos hábitos y conductas, implica fuentes de apoyo importantes para el paciente (pareja, familia, trabajo) y ayuda a delimitar el tipo de objetivos por establecer (tanto terapéuticos como vitales). Por estas razones, sería interesante disponer de un instrumento que, a diferencia de otras medidas como las escalas diagnósticas, las escalas de gravedad sintomática o los instrumentos de calidad de vida, permitiera cuantificar el nivel de OV de la población que demanda atención psicológica. Este sería útil para la toma de decisiones clínicas, y permitiría determinar la viabilidad de aplicar directamente los tratamientos psicológicos empíricamente validados (TEA) (Labrador \& Crespo, 2012) o intervenir en primer lugar sobre algún aspecto de la $\mathrm{OV}$, para aumentar la eficacia de los TEA.

Con el propósito de delimitar la OV y desarrollar una evaluación de esta, se ha diseñado el presente trabajo, cuyos objetivos específicos son: (1) describir la OV de una muestra clínica en el momento en el que demandan atención psicológica, (2) examinar la relación entre variables de OV y variables clínicas y (3) examinar la relación entre variables de OV y la eficacia del tratamiento (proceso y resultados). Se pretende de esta forma identificar en qué medida la presencia de una mayor o menor OV se relaciona con variables de gravedad clínica y con el aprovechamiento y éxito de los programas de tratamiento.

\section{Método}

\section{Participantes}

Fueron 78 pacientes del Servicio Ambulatorio de Atención Psicológica del Máster de Psicología Clínica y de la Salud de la UCM (MPCS). El 59 \% de la muestra eran mujeres y la edad media 35.65 años $(D E=12.63)$. Los trastornos más frecuentes fueron los trastornos de ansiedad (50\%) y un $41 \%$ de la muestra presentaba comorbilidad. En la Tabla 1 se muestra el promedio de diagnósticos en los Ejes I-IV de la muestra y la puntuación media en la EEAG de la clasificación multiaxial DSM-IV-TR (APA, 2000).

El $61.5 \%$ finalizó con éxito el tratamiento, en el $51.3 \%$ de los casos se estimó un porcentaje de recuperación entre el 76 y el $100 \%$ y en un $70.5 \%$ no hubo recaídas. En la Tabla 2 se indica el promedio de sesiones, de objetivos y de técnicas empleadas en el tratamiento.

\section{TABLA 1}

Número de diagnósticos clínicos y promedio de la actividad global de la muestra

\begin{tabular}{lccc}
\hline Ejes DSM-IV-TR & Media & DE & Rango \\
\hline Eje I & 1.31 & 0.76 & $0-4$ \\
Eje II & 0.17 & 0.4 & $0-2$ \\
Eje III & 0.51 & 0.76 & $0-3$ \\
Eje IV & 1.36 & 1.12 & $0-6$ \\
Eje V (EEAG) & 64.31 & 9.92 & $40-85$ \\
\hline
\end{tabular}

Fuente: elaboración propia

TABLA 2

Promedio de sesiones, objetivos y técnicas de tratamiento de la muestra

\begin{tabular}{lccc}
\hline Variables de tratamiento & Media & DE & Rango \\
\hline Sesiones & 27.82 & 17.77 & $0-88$ \\
Objetivos terapéuticos & 8.1 & 2.73 & $3-18$ \\
Técnicas empleadas & 13.82 & 4.78 & $4-30$
\end{tabular}

Fuente: elaboración propia 


\section{Variables e instrumentos}

Las variables sociodemográficas se registraron en el instrumento de acceso a la unidad del MPCS. Las variables clínicas fueron el número de diagnósticos en los Ejes I-IV y la puntuación en el Eje V (EEAG) del DSM-IV-TR (APA, 2000). Los pacientes fueron evaluados siguiendo los criterios diagnósticos del DSM-IV-TR, con los instrumentos apropiados en cada caso (entrevista clínica, cuestionarios y autorregistros).

Las variables de tratamiento fueron: número de sesiones, número de objetivos y técnicas recogidas en el plan de tratamiento, resultado de tratamiento (alta o abandono), presencia de recaídas (el paciente vuelve al mismo estado que le llevó a consulta una vez conseguidos los objetivos terapéuticos pertinentes) y grado de cumplimiento de los objetivos terapéuticos. Estas variables fueron registradas en los historiales clínicos.

Las variables de OV se codificaron mediante una escala heteroaplicada, confeccionada ad hoc para esta investigación, denominada Escala de Organización Vital (EOV). Un equipo de 17 terapeutas elaboró la escala inicialmente, y se contó con la consideración de otros 84 terapeutas para establecerla definitivamente. La EOV está compuesta por 23 ítems divididos en 6 áreas de organización: personal, familiar, pareja, laboral, social y coterapeuta. Cada uno de los ítems se puntúa de forma dicotómica indicando 1: presencia y 0 : ausencia de la característica evaluada, a excepción del último ítem que puntúa 3, en el caso de contar con una persona del entorno que pueda participar en el tratamiento como coterapeuta. De este modo, se obtiene una puntuación específica de cada área y

TABLA 3

Propuesta de la escala de organización vital (EOV)

\begin{tabular}{|c|c|c|c|c|}
\hline Áreas & Ítems & No & Sí & Total \\
\hline \multirow{6}{*}{ Personal } & Buenos hábitos de alimentación & 0 & 1 & \multirow{6}{*}{16} \\
\hline & Buena higiene & 0 & 1 & \\
\hline & Sueño reparador & 0 & 1 & \\
\hline & Práctica de ejercicio físico & 0 & 1 & \\
\hline & Horarios regulares & 0 & 1 & \\
\hline & Ausencia de consumo de sustancias & 0 & 1 & \\
\hline \multirow{4}{*}{ Familiar } & Tiene familia & 0 & 1 & \multirow{4}{*}{$/ 4$} \\
\hline & Vive con familia & 0 & 1 & \\
\hline & Calidad de relación con familia & 0 & 1 & \\
\hline & Apoyo percibido por el paciente de su familia & 0 & 1 & \\
\hline \multirow{4}{*}{ Pareja } & Tiene pareja & 0 & 1 & \multirow{4}{*}{$/ 4$} \\
\hline & Convive con su pareja & 0 & 1 & \\
\hline & Calidad de la relación con su pareja & 0 & 1 & \\
\hline & Apoyo percibido por el paciente de su pareja & 0 & 1 & \\
\hline \multirow{4}{*}{ Laboral } & Tiene una ocupación & 0 & 1 & \multirow{4}{*}{$/ 4$} \\
\hline & Ocupación estable & 0 & 1 & \\
\hline & Satisfacción con esta ocupación & 0 & 1 & \\
\hline & Entorno laboral de apoyo & 0 & 1 & \\
\hline \multirow{4}{*}{ Social } & Cuenta con grupo de amigos & 0 & 1 & \multirow{4}{*}{$/ 4$} \\
\hline & Pertenece a más de un grupo social & 0 & 1 & \\
\hline & Apoyo social percibido & 0 & 1 & \\
\hline & Ocio de calidad & 0 & 1 & \\
\hline Coterapeuta & Persona del entorno que podría estar implicada en el tratamiento & 0 & 3 & 13 \\
\hline
\end{tabular}

Fuente: elaboración propia 
una puntuación total. El rango de la puntuación total oscila entre 0 y 25 puntos. Puntuaciones más altas indicarían una mayor OV. El análisis de consistencia interna global de la EOV, mediante el coeficiente alpha de Cronbach, fue 0.72. La estructura de la escala aparece en la Tabla 3.

\section{Procedimiento}

La muestra se obtuvo seleccionando al azar de la base de datos de la unidad del MPCS, 78 casos atendidos entre los años 2009-2011. Se excluyeron menores de edad y aquellos en los que la información estuviera incompleta.

Las variables clínicas, de tratamiento y las variables incluidas en la EOV fueron extraídas de los historiales clínicos y de los diarios de sesión de cada paciente, una vez finalizada su relación terapéutica con la unidad del MPCS. En cada caso, dos terapeutas registraron las variables de interés y puntuaron la EOV, uno de ellos era el propio terapeuta del paciente y otro un terapeuta sin relación con el caso. Para comprobar la fiabilidad del método de evaluación de los 78 casos de la muestra, se procedió a hacer un análisis de fiabilidad interjueces con 32 de estos casos, obteniendo una puntuación de 0.84 .

\section{Análisis de datos}

Se realizaron análisis descriptivos y de frecuencias para describir las características sociodemográficas, clínicas y de tratamiento de la muestra, así como para analizar su OV. Para analizar la relación entre las variables clínicas y de tratamiento con las variables de OV, se utilizó el coeficiente de correlación de Pearson. Para completar el análisis correlacional,

\section{TABLA 4}

Resultados de la muestra en los ítems de la escala de organización vital (EOV)

\begin{tabular}{llc}
\hline Áreas EOV & Ítems EOV & $\%$ \\
\hline \multirow{4}{*}{ Personal } & Buenos hábitos de alimentación & 82.1 \\
& Buena higiene & 94.9 \\
& Sueño reparador & 50 \\
& Práctica de ejercicio físico & 33.3 \\
& Horarios regulares & 70.5 \\
Familiar & Ausencia de consumo de sustancias & 82.1 \\
& Tiene familia & 100 \\
& Vive con familia & 70.5 \\
& Calidad de relación con familia & 56.4 \\
Pareja & Apoyo percibido por el paciente de su familia & 75.6 \\
& Tiene pareja & 56.4 \\
& Convive con su pareja & 38.5 \\
& Calidad de la relación con su pareja & 20.5 \\
Laboral & Apoyo percibido por el paciente de su pareja & 41 \\
& Tiene una ocupación & 79.5 \\
& Ocupación estable & 66.7 \\
& Satisfacción con esta ocupación & 51.3 \\
Social & Entorno laboral de apoyo & 33.3 \\
\hline Coterapeuta & Cuenta con grupo de amigos & 78.2 \\
& Pertenece a más de un grupo social & 33.3 \\
\hline & Apoyo social percibido & 57.7 \\
& Ocio de calidad & 44.9 \\
\hline
\end{tabular}

Fuente: elaboración propia 
Francisco J. Labrador, Gloria García-Fernández, Mónica Bernaldo-de-Quirós, Roberto Gómez, Cristina Méndez, Ignacio Fernández-Arias

TABLA 5

Puntuaciones medias de la muestra en las áreas de la escala de organización vital (EOV)

\begin{tabular}{lccc}
\hline Áreas EOV & Media & DE & Rango \\
\hline Personal & 4.1 & 1.21 & $1-6$ \\
Familiar & 3.01 & 0.9 & $1-4$ \\
Pareja & 1.6 & 152 & $0-4$ \\
Laboral & 2.33 & 1.38 & $0-4$ \\
Social & 2.1 & 1.43 & $0-4$ \\
\hline Puntuación Total EOV & 15.54 & 4.29 & $6-24$ \\
\hline
\end{tabular}

Fuente: elaboración propia

se compararon las puntuaciones de OV de la muestra en función del resultado de tratamiento (alta o abandono) y se generaron dos grupos de sujetos con puntuaciones totales extremas en la EOV.

En concreto, se fijó el percentil 25 y el percentil 75 como puntos de corte que definían los sujetos con menor y mayor OV, respectivamente. Con cada una de las agrupaciones obtenidas, se hicieron comparaciones de las variables de tratamiento utilizando la prueba $t$ de Student y la prueba $\chi^{2}$. Se aplicó la corrección de Bonferroni para comparaciones múltiples con el objetivo de evitar la comisión del error Tipo I. Todos los datos fueron analizados utilizando el paquete estadístico SPSS19.

\section{Resultados}

\section{Descripción de la organización vital de la muestra clínica}

En la Tabla 4 aparece el porcentaje de sujetos que presentan cada uno de los ítems de la EOV y en la Tabla 5 se presentan las puntuaciones medias de la muestra en cada área.

Los resultados indican que las áreas con puntuaciones más elevadas son la personal y la familiar. En la mayoría de los casos, se observan unos buenos hábitos de alimentación (82.1\%) e higiene (94.9\%), así como una ausencia de consumo de sustancias $(82.1 \%)$. Igualmente, un alto porcentaje de la muestra (70.5\%) mantiene rutinas en su día a día (horarios de comida, sueño, trabajo, etc.). Solo la mitad de los pacientes consigue tener un sueño reparador $(50 \%)$ y un tercio practica algún tipo de ejercicio físico de forma habitual (33.3 \%). Destaca que la totalidad de la muestra tiene familia (100\%), aunque solo el $56.4 \%$ de los casos considera de calidad la relación familiar. En el área de pareja, el $56.4 \%$ de la muestra tiene pareja y solo un $20.5 \%$ percibe calidad en la relación.

En el área laboral, el $79.5 \%$ tiene una ocupación, siendo estable en un $66.7 \%$ de los casos. Sin embargo, únicamente la mitad (51.3 \%) están satisfechos con su situación laboral y solo un tercio (33.3 \%) desarrolla su actividad profesional en un entorno de apoyo.

En el ámbito social, un $78.2 \%$ de los pacientes cuenta con un grupo de amigos y además el $33.3 \%$ dispone de más de un grupo (compañeros de trabajo, de actividades deportivas, etc.). Sin embargo, solo el $57.7 \%$ de los casos percibe apoyo social. En cuanto al ocio, menos de la mitad de los casos (44.9\%) lo consideran satisfactorio. Finalmente, el $79.5 \%$ dispone de alguna persona de su entorno cercano para participar como coterapeuta.

\section{Relación entre organización vital y variables diagnósticas}

En la Tabla 6 se muestra la relación entre variables diagnósticas (número de diagnósticos en los Ejes IIV y puntuación en el Eje V del DSM-IV-TR) y las puntuaciones obtenidas en la EOV.

Los resultados indican que un mayor número de enfermedades médicas (Eje III) se relaciona con una peor organización en el área de pareja ( $r x y-0.365 ; p$ $<0.001$ ) y la correlación entre el área familiar y el Eje II ( $r x y-0.322 ; p=0.004$ ) indica una tendencia de una mayor presencia de diagnósticos en el Eje II asociada a una peor organización familiar. 
TABLA 6

Correlaciones de las puntuaciones de la escala de organización vital y variables diagnósticas

\begin{tabular}{|c|c|c|c|c|c|c|c|c|c|c|}
\hline & \multicolumn{2}{|c|}{ Eje I } & \multicolumn{2}{|c|}{ Eje II } & \multicolumn{2}{|c|}{ Eje III } & \multicolumn{2}{|c|}{ Eje IV } & \multicolumn{2}{|c|}{ Eje V } \\
\hline & $\mathrm{r}$ & $\mathrm{p}$ & $\mathrm{r}$ & $\mathrm{p}$ & $\mathrm{r}$ & $\mathrm{p}$ & $\mathrm{r}$ & $\mathrm{r}$ & $\mathrm{r}$ & $\mathrm{p}$ \\
\hline Áreas EOV & & & & & & & & & & \\
\hline $\begin{array}{l}\text { Per } \\
\text { Far }\end{array}$ & .063 & $\begin{array}{l}0.585 \\
0.229\end{array}$ & $\begin{array}{l}-0.114 \\
-0.322\end{array}$ & 0.322 & 0.18 & 0.115 & 0.191 & $\begin{array}{l}0.094 \\
0.75\end{array}$ & $\begin{array}{l}0.292 \\
0.100\end{array}$ & 0.01 \\
\hline & .00 & 0.9 & -0.164 & & -0.3 & 0.00 & $\begin{array}{l}-0.132 \\
-0.09\end{array}$ & $\begin{array}{l}0.2 \\
0.4\end{array}$ & $\begin{array}{l}0.199 \\
0.083\end{array}$ & 0.471 \\
\hline $\mathrm{Lab}$ & 1 & 0.1 & -08 & 0.9 & 0.1 & 0.303 & -0.111 & 0.3 & 0.31 & $0.006^{*}$ \\
\hline Socia & .0 & 0.5 & -0.184 & 0.1 & 0.0 & 0.6 & -0.143 & 0.211 & 0.51 & $0.001 * *$ \\
\hline Total EOV & -0.107 & 0.351 & -0.274 & 0.01 & 0.242 & 0.033 & -0.124 & 0.281 & 0.493 & $0.001 * *$ \\
\hline
\end{tabular}

$* *_{p}<0.0016 . *_{p}<0.01$.

Fuente: elaboración propia

Por otro lado, se observa cómo las puntuaciones en la EEAG del Eje $\mathrm{V}$ se relacionan significativamente con el área social ( $r x y 0.510 ; p<0.001)$ y la puntuación total de la escala EOV ( $r x y 0.493 ; p<$ 0.001). Estas correlaciones son positivas y relativamente elevadas. Además, se observa una tendencia en la asociación entre el área laboral de la EOV y las puntuaciones en el Eje V $(r x y 0.310 ; p=0.006)$.

\section{Relación entre organización vital} $y$ variables de tratamiento

En la Tabla 7 se muestra la relación entre las puntuaciones obtenidas en la EOV y en la EEAG y las variables relacionadas con el tratamiento.

En especial destaca la correlación estadísticamente significativa entre el área personal de la EOV y el número de técnicas del plan de tratamiento $($ rxy - 0.386; $p<0.001)$. Parece que una mayor organización personal implica un menor número de técnicas en el plan de tratamiento. Además, se muestra una tendencia $(p<0.01)$ a relacionar el número de objetivos de tratamiento con el área social y con la puntuación total en la EOV. La dirección es siempre la misma, a mayor OV menor número de objetivos terapéuticos.

En la Tabla 8 se compara la OV de la muestra en base al resultado final del tratamiento. Tal como se observa, no hay diferencias estadísticamente significativas en las variables de OV entre pacientes que abandonan o finalizan con éxito el tratamiento, aunque la tendencia en todas las medidas es que los que han abandonado tienen peores puntuaciones en su OV.

Además, en la Tabla 9 se comparan las variables de tratamiento de los grupos extremos de OV, generados a partir de los cuartiles 1 y 3 . Los resultados mostraron diferencias estadísticamente significa-

TABLA 7

Correlaciones de las escalas de organización vital y de evaluación de la actividad global y las variables de tratamiento

\begin{tabular}{|c|c|c|c|c|c|c|c|c|c|c|c|c|}
\hline & \multicolumn{2}{|c|}{ Sesiones } & \multicolumn{2}{|c|}{ Objetivos } & \multicolumn{2}{|c|}{ Técnicas } & \multicolumn{2}{|c|}{$\begin{array}{l}\text { Grado de } \\
\text { cumplimiento }\end{array}$} & \multicolumn{2}{|c|}{ Recaídas } & \multicolumn{2}{|c|}{$\begin{array}{l}\text { Resultado del } \\
\text { tratamiento }\end{array}$} \\
\hline & $\mathrm{r}$ & $\mathrm{p}$ & $\mathrm{r}$ & $\mathrm{p}$ & $\mathrm{r}$ & $\mathrm{p}$ & $\mathrm{r}$ & $\mathrm{p}$ & $\mathrm{r}$ & $\mathrm{p}$ & $\mathrm{r}$ & $\mathrm{p}$ \\
\hline \multicolumn{13}{|l|}{ Áreas } \\
\hline Personal & 0.23 & 0.042 & -0.289 & 0.01 & -0.386 & $<0.001 * *$ & 0.162 & 0.158 & 0.148 & 0.195 & 0.045 & 0.693 \\
\hline Familiar & 0.111 & 0.334 & 0.089 & 0.44 & 0.106 & 0.357 & 0.084 & 0.465 & 0.041 & 0.725 & 0.099 & 0.387 \\
\hline Pareja & 0.024 & 0.834 & -0.221 & 0.052 & -0.179 & 0.116 & 0.111 & 0.334 & 0.183 & 0.108 & 0.054 & 0.641 \\
\hline Laboral & -0.195 & 0.087 & -0.184 & 0.107 & -0.154 & 0.179 & 0.065 & 0.571 & 0.136 & 0.234 & 0.077 & 0.505 \\
\hline Social & -0.181 & 0.113 & -0.352 & $0.002 *$ & -0.171 & 0.134 & 0.068 & 0.551 & -0.013 & 0.913 & 0.057 & 0.622 \\
\hline Total & -0.013 & 0.913 & -0.35 & $0.002 *$ & -0.246 & 0.03 & 0.183 & 0.109 & 0.161 & 0.16 & 0.112 & 0.328 \\
\hline Eje V & -0.159 & 0.163 & -0.177 & 0.121 & -0.238 & 0.036 & 0.101 & 0.381 & -0.045 & 0.693 & 0.11 & 0.337 \\
\hline
\end{tabular}

$* * \mathrm{p}<0.0011 * \mathrm{p}<0.01$

Fuente: elaboración propia 
Francisco J. Labrador, Gloria García-Fernández, Mónica Bernaldo-de-Quirós, Roberto Gómez, Cristina Méndez, Ignacio Fernández-Arias

TABLA 8

Comparación de la organización vital inicial de la muestra en función del resultado del tratamiento

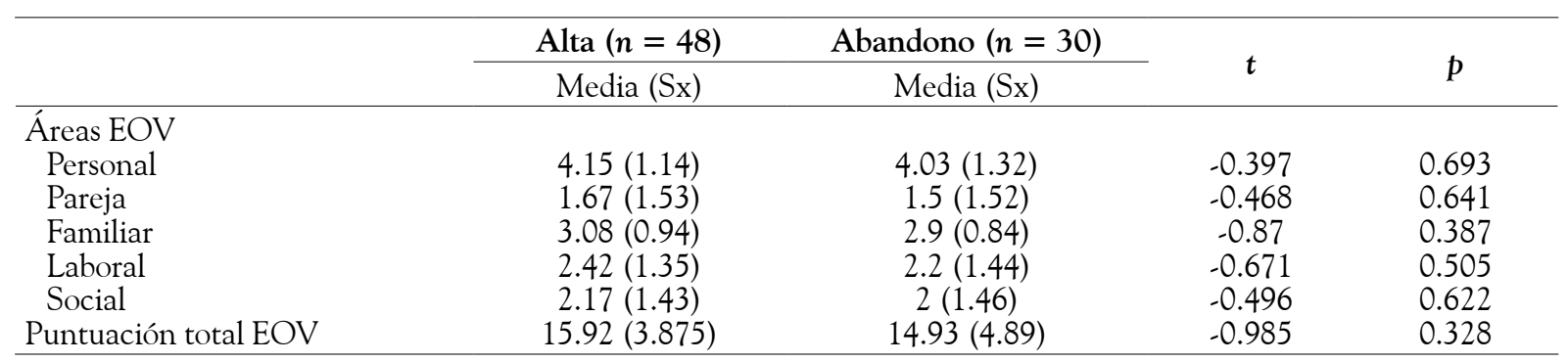

Fuente: elaboración propia

TABLA 9

Comparación de los resultados de tratamiento en función del nivel de organización vital

\begin{tabular}{lcccl}
\hline & $\begin{array}{c}\text { OV baja } \leq 13 \\
(\boldsymbol{n}=20)\end{array}$ & $\begin{array}{c}\text { OV alta } \geq 19 \\
(\boldsymbol{n}=20)\end{array}$ & $\boldsymbol{t} / \chi^{2}$ & \multicolumn{1}{c}{$\boldsymbol{p}$} \\
\hline Sesiones & $27.9(20.23)$ & $27.8(15.92)$ & 0.017 & 0.986 \\
Objetivos & $9.6(2.76)$ & $6.85(1.92)$ & 3.653 & $0.001^{*}$ \\
Técnicas & $15.3(4.56)$ & $12.15(4.52)$ & 2.192 & 0.035 \\
Alto cumplimiento terapéutico (\%) & 35 & 55 & 2.489 & 0.477 \\
Ausencia de recá́das (\%) & 60 & 75 & 1.026 & 0.311 \\
Altas terapéuticas (\%) & 50 & 70 & 1.667 & 0.197 \\
\hline
\end{tabular}

$* p<0.008$.

Fuente: elaboración propia

tivas en el número de objetivos de tratamiento de ambos grupos $(t=3.653 ; p=0.001)$. El grupo de menor OV presenta un mayor número de objetivos en el plan de tratamiento en comparación con el grupo de mayor OV.

\section{Discusión}

El interés del presente estudio radica en el intento de caracterizar el concepto de OV y evaluar su relevancia de cara a la intervención en psicología clínica. Las consideraciones intuitivas provenientes de la práctica clínica apuntaban la necesidad de un nivel mínimo de OV para proceder al tratamiento. Este concepto de OV ha de operativizarse y en este trabajo se han definido alrededor de 5 subáreas (personal, pareja, familiar, laboral y social), más un dato específico de cara a la intervención: presencia de un coterapeuta. A partir de esta conceptualización, se ha creado la escala EOV que orientaría de forma precisa en qué dirección trabajar para mejorar la OV o aseguraría dónde se presentan ya niveles adecuados.

A partir de los datos obtenidos, se establece además un perfil de OV de referencia de la población que demanda atención psicológica, en la actualidad. Estos resultados ayudan a identificar áreas de funcionamiento críticas y necesidades, así como a diseñar intervenciones. Por ejemplo, considerando la primera de las áreas: personal, parece que en general no hay problemas con los hábitos de alimentación e higiene, sin embargo, aparecen dificultades con el sueño reparador (50\%) o con el ejercicio físico, pues solo un $33.3 \%$ lo realiza. En este sentido, estudios previos han enfatizado cómo la prescripción de ejercicio físico es útil para prevenir la ansiedad y la depresión (Subirats, Subirats-Vila, \& Soteras-Martínez, 2012). Además, los trastornos del sueño se han relacionado habitualmente con los trastornos de ansiedad en los que la pérdida de sueño puede mantener estos cuadros; sin embargo, y a pesar de que el tratamiento para los trastornos 
de ansiedad y el insomnio tienen muchos componentes que se superponen, la secuencia óptima de las pautas sigue estando poco investigada (Mellman, 2006). Estos datos alertan sobre la necesidad de evaluar precozmente el sueño y el ejercicio físico para diseñar el plan de tratamiento.

También llama la atención que casi la mitad de los pacientes no tiene pareja (43.6\%), y el hecho de que solo el $20.5 \%$ perciban calidad en esa relación, o que solo el 33 \% tenga más de un grupo de amigos de referencia. De hecho, de todas las áreas, es el área de la pareja en la que los pacientes obtienen unas puntuaciones inferiores (1.6 sobre 4), la única por debajo de 2, mientras que el área personal (4.1 sobre 6) y familiar (3.01 sobre 4) son las que aparecen con puntuaciones más elevadas. Estos resultados se corresponden con los datos de la última Encuesta Nacional de Salud de España (Bones Rocha et al., 2010), en la que se encontró que entre las variables más asociadas a una mayor prevalencia de problemas de salud mental está el hecho de estar separado o divorciado y tener poco apoyo social.

Otro aspecto importante es analizar hasta qué punto la OV se relaciona con las variables diagnósticas. Las correlaciones entre las puntuaciones de la EOV y los distintos ejes del DSM-IV-TR, han alcanzado en algunos casos valores elevados (alrededor del 0.5) y significativos. La más importante, sin duda, es la correlación encontrada entre el eje V y la puntuación total de la EOV. Ciertamente, el objetivo del eje $\mathrm{V}$ y de la $\mathrm{EOV}$ es próximo, pero frente a una evaluación eminentemente subjetiva y general del eje $\mathrm{V}$, la evaluación de la EOV es más precisa e incluye tanto información general como específica por áreas.

Las subáreas de la EOV que correlacionan con el Eje V son las áreas social y laboral, mientras que las áreas personal, familiar y de pareja no correlacionan de forma significativa, quizá debido a que no son por definición evaluadas en la EEAG (APA, 2000). Además, el hecho de no encontrar correlaciones significativas en la mayoría de los Ejes I-IV y la EOV puede indicar que efectivamente la EOV no se solapa con la evaluación puramente sintomática de los Ejes I-IV. De este modo, supera una de las críticas más extendidas en el uso de la EEAG en el que la evaluación sintomática y psicosocial se solapan (Goldman et al., 1992; Smith et al., 2011).

En cuanto a la relación entre las puntuaciones de la EOV y las variables relacionadas con el tratamiento, destaca que la organización personal alcanza una correlación significativa (alrededor de 0.4) con el número de técnicas de tratamiento, indicando que cuanto mayores son las puntuaciones en este área menos técnicas se han utilizado. Ya en trabajos anteriores se señaló que una variable de especial relevancia para prolongar los tratamientos era el número de técnicas (Bernaldo-de-Quirós, Labrador, Estupiñá, \& Fernández-Arias, 2013; Labrador, Bernaldo-de-Quirós, \& Estupiñá, 2011), en consecuencia, esta relación negativa entre $\mathrm{OV}$ y número de técnicas puede ser interesante para reducir la duración del tratamiento.

Sin embargo, no aparecen correlaciones entre la OV y el resultado final del tratamiento o las recaídas, tampoco al analizar las diferencias entre las medias de los pacientes que han logrado el alta terapéutica y los que no. Aunque es de destacar que la tendencia es constante, todas las puntuaciones de la EOV del grupo de pacientes que han logrado el alta son superiores a las del grupo de abandono. Con todo, las puntuaciones promedio tanto del grupo de altas (15.92), como del grupo de abandonos (14.93), son más bien elevadas, lo que implica que probablemente la mayoría de los pacientes tenían ya una OV importante. Por otro lado, al comparar los casos de los pacientes con puntuaciones más extremas, por debajo de 13 y por encima de 19, se observa cómo los pacientes de baja OV requieren un mayor número de objetivos en su plan de tratamiento y un mayor porcentaje abandona el tratamiento, sufren más recaídas y muestran un menor aprovechamiento de la intervención.

No obstante, este trabajo inicial de corte exploratorio y orientativo no está exento de limitaciones. La primera referida a la falta de tipificación del instrumento. Por otro lado, es probable que con una muestra más amplia pudieran aparecer resultados más significativos y la selección al azar quizás no sea suficiente para garantizar una adecuada representación de la demanda clínica 
actual, aunque la representación de los Trastornos de Ansiedad es próxima a otras muestras (Haro et al., 2006; Kessler, Chiu, Demler, Merikangas, \& Walters, 2005). El hecho de que la evaluación con la EOV se haya llevado a cabo por parte del propio terapeuta y a la vez por un terapeuta que desconoce al paciente, solo a partir de los datos de la historia clínica, obteniéndose un índice de acuerdo interjueces de 0.84 , pone de relieve, por un lado, un alto grado de fiabilidad y, por otro, la facilidad de su aplicación. Sin embargo, la codificación a partir de las historias de los pacientes puede no ser la mejor estrategia y probablemente recoger esta información directamente del paciente permitiría una mayor precisión. Finalmente, hubiera sido interesante evaluar la OV al final del tratamiento y tener los datos de OV de personas sin problemas psicológicos como criterios de referencia. Este es un nuevo trabajo que está ya en marcha, pero que excede el objetivo del presente.

En resumen, este es un estudio preliminar para encaminar futuras líneas de investigación relacionadas con la creación de instrumentos para la evaluación de características psicosociales relevantes en la toma de decisiones clínicas y en la medición de la efectividad de los tratamientos psicológicos. Parece una vía interesante de trabajo, no absolutamente novedosa, pero que puede aportar información de relevancia para el psicólogo clínico y con gran facilidad y rapidez. Los resultados, aunque limitados, animan a seguir con esta tarea y sin duda habrá que trabajar en la precisión del instrumento y en la forma y momentos de aplicación.

\section{Referencias}

Alonso, J., \& Lepine, J. P. (2007). Overview of key data from the European Study of the Epidemiology of Mental Disorders (ESEMeD). Journal of Clinical Psychiatry, 68(Supl. 2), 3-9.

American Psychiatric Association. (2000). Diagnostic and statistical manual of mental disorders (4.a ed. rev.). Washington, DC: American Psychiatric Association.

Bernaldo-de-Quirós, M., Labrador, F. J., Estupiñá, F. J., \& Fernández-Arias, I. (2013). La duración de los tratamientos psicológicos: diferencias entre los casos de corta, media y larga duración. Universitas Psychologica, 12(1), 21-30.

Bones Rocha, K., Pérez, K., Rodríguez-Sanz, M., Borrell, C., \& Obiols, J. E. (2010). Prevalencia de problemas de salud mental y su asociación con variables socioeconómicas, de trabajo y salud: resultados de la Encuesta Nacional de Salud de España. Psicothema, 22(3), 389-395.

Dimsdale, J. E., Jeste, D. V., \& Patterson, T. L. (2010). Beyond the global assessment of functioning: Learning from Virginia Apgar. Psychosomatics, 51(6), 515-519.

Fava, G. A., Rafanelli, C., \& Tomba, E. (2012). The clinical process in psychiatry: A clinimetric approach. The Journal of Clinical Psychiatry, 73(2), 177-184.

García-Portilla, M. P., Saiz, P. A., Bousoño, M., Bascaran, M. T., Guzman-Quilo, C., \& Bobes, J. (2011). Validación de la versión española de la escala de Funcionamiento Personal y Social en pacientes ambulatorios con esquizofrenia estable e inestable. Revista de Psiquiatria y Salud Mental, 4(1), 9-18.

Goldman, H. H., Skodol, A. E., \& Lave, T. R. (1992). Revising axis $\mathrm{V}$ for DSM-IV: A review of measures of social functioning. The American Journal of Psychiatry, 149(9), 1148-1156.

Haro, J. M., Palacín, C., Vilagut, G., Martínez, M., Bernal, M., Luque, I., ... Alonso, J. (2006). Prevalencia de los trastornos mentales y factores asociados: resultados del estudio ESEMeD-España. Medicina Clínica, 126(12), 445-451.

Hilsenroth, M. J., Ackerman, S. J., Blagys, M. D., Baumann, B. D., Baity, M. R., Smith, S. R., ... Holdwick, D. J. (2000). Reliability and validity of DSMIV axis V. The American Journal of Psychiatry, 157(11), 1858-1863.

Kessler, R. C., Chiu, W. T., Demler, O., Merikangas, K. R., \& Walters, E. E. (2005). Prevalence, severity, and comorbidity of 12-month DSM-IV disorders in the National Comorbidity Survey Replication. Archives of General Psychiatry, 62(6), 617-627.

Labrador, F. J., \& Crespo, M. (2012). Psicología clínica basada en la evidencia. Madrid: Pirámide.

Labrador, F. J., Bernaldo-de-Quirós, M., \& Estupiñá, F. J. (2011). ¿Por qué se alargan los tratamientos psicológicos? Predictores de una mayor duración 
del tratamiento y diferencias entre los casos de corta y larga duración. Psicothema, 23(4), 573-579.

Lam, R. W., Filteau, M. -J., \& Milev, R. (2011). Clinical effectiveness: The importance of psychosocial functioning outcomes. Journal of Affective Disorders, 132, S9-S13.

Lozano, O. M., Rojas, A. J., Perez, C., González-Sáiz, F., Ballesta, R., \& Izaskun, B. (2008). Evidencias de validez del test para la evaluación de la calidad de vida en adictos a sustancias psicoactivas a partir del modelo biaxial de la adicción. Psicothema, 20(2), 317-323.

Lucas-Carrasco, R. (2012). The WHO quality of life (WHOQOL) questionnaire: Spanish development and validation studies. Quality of Life Research, 21(1), 161-165.

McKnight, P. E., \& Kashdan, T. B. (2009). The importance of functional impairment to mental health outcomes: A case for reassessing our goals in depression treatment research. Clinical Psychology Review, 29(3), 243-259.

Mellman, T. A. (2006). Sleep and anxiety disorders. The Psychiatric Clinics of North America, 29(4), 1047-1058.

Morosini, P. L., Magliano, L., Brambilla, L., Ugolini, S., \& Pioli, R. (2000). Development, reliability and acceptability of a new version of the DSM-IV Social and Occupational Functioning Assessment Scale (SOFAS) to assess routine social functioning. Acta Psychiatrica Scandinavica, 101(4), 323-329.

Mundo, E., Bonalume, L., Del Corno, F., Madeddu, F., \& Lang, M. (2010). Kennedy V axis assessment in an Italian outpatient and inpatient population. Rivista Di Psichiatria, 45(4), 214-220.

Niv, N., Cohen, A. N., Sullivan, G., \& Young, A. S. (2007). The MIRECC version of the global assessment of functioning scale: Reliability and validity. Psychiatric Services, 58(4), 529-535.

Rafanelli, C., \& Ruini, C. (2012). Assessment of psychological well-being in psychosomatic medicine. Advances in Psychosomatic Medicine, 32, 182-202.

Rebollo, P. (2008). Utilización del "grupo SF" de los cuestionarios de calidad de vida (SF-36, SF-12 and SF-6D) en el marco de ensayos clínicos y en la práctica clínica habitual. Medicina Clinica, 130(19), 740-741.

Ritsner, M. S., Arbitman, M., Lisker, A., \& Ponizovsky, A. M. (2012). Ten-year quality of life outcomes among patients with schizophrenia and schizoaffective disorder II. Predictive value of psychosocial factors. Quality of Life Research, 21(6), 1075-1084.

Sánchez-Hervás, E., Santonja Gómez, F. J., Secades Villa, R., García-Fernández, G., García-Rodriguez, O., \& Zacares Romaguera, F. (2012). Psychosocial predictors of relapse in cocaine-dependent patients in treatment. The Spanish Journal of Psychology, 15(2), 748-755.

Silveira, M., Kern, E., \& Chem, C. (2012). Qualidade de vida e auto-imagem de pacientes com câncer de cabeça e pescoço. Universitas Psychologica, 11(1), 13-23.

Smith, G. N., Ehmann, T. S., Flynn, S. W., MacEwan, G. W., Tee, K., Kopala, L. C., ... Honer, W. G. (2011). The assessment of symptom severity and functional impairment with DSM-IV Axis V. Psychiatric Services, 62(4), 411-417.

Subirats, E., Subirats-Vila, G., \& Soteras-Martínez, I. (2012). Prescripción de ejercicio físico: indicaciones, posología y aspectos adversos. Medicina Clinica, 138(1), 18-24.

Tracy, E. M., Laudet, A. B., Min, M. O., Kim, H., Brown, S., Jun, M. K., \& Singer, L. (2012). Prospective patterns and correlates of quality of life among women in substance abuse treatment. Drug and Alcohol Dependence, 124(3), 242-249.

Urzúa, A., \& Caqueo-Urízar, A. (2012). Calidad de vida: una revisión teórica del concepto. Terapia Psicológica, 30(1), 61-71.

Velarde-Jurado, E., \& Ávila-Figueroa, C. (2002a). Evaluación de la Calidad de Vida. Salud Pública de México, 44(4), 349-361.

Velarde-Jurado, E., \& Ávila-Figueroa, C. (2002b). Consideraciones metodológicas para evaluar la calidad de vida. Salud Pública de México, 44(5), 448-463.

Verdonck-de Leeuw, I. M., van Nieuwenhuizen, A., \& Leemans, C. R. (2012). The value of quality-of-life questionnaires in head and neck cancer. Current Opinion in Otolaryngology $\mathbb{E}$ Head and Neck Surgery, 20(2), 142-147. 
